\title{
Vergleichende Untersuchung der Zentrale-Orte-Konzepte in den Ländern und Empfehlungen zu ihrer Weiterentwicklung
}

\author{
Stefan Greiving • Florian Flex $\cdot$ Thomas Terfrüchte
}

Eingegangen: 19. Dezember 2014 / Angenommen: 13. Juli 2015 / Online publiziert: 31. Juli 2015

(C) Springer-Verlag Berlin Heidelberg 2015

Zusammenfassung Zentrale-Orte-Konzepte sind fester Bestandteil aller Pläne und Programme der Landes- und Regionalplanung der Flächenländer. Gerade im Zusammenhang mit den Herausforderungen, die aus dem demographischen Wandel für die Sicherung der Daseinsvorsorge erwachsen, wird den Zentrale-Orte-Konzepten eine zunehmende Bedeutung beigemessen. Wesentliche Ziele des Beitrags sind die Darstellung und Analyse des Status quo der Zentrale-Orte-Konzepte in den Ländern sowie Empfehlungen zu ihrer Weiterentwicklung.

Im Ergebnis der Analyse aller Pläne und Programme der Flächenländer ist festzuhalten, dass es das ZentraleOrte-Konzept nicht mehr gibt. Vielmehr haben sich unterschiedliche Steuerungskonzepte in den Ländern entwickelt. Damit einhergegangen ist auch ein unterschiedliches Begriffsverständnis hinsichtlich der elementaren Bestandteile von Zentrale-Orte-Konzepten wie Schwellenwerte für Tragfähigkeit und Erreichbarkeit, Hierarchiestufen und Ausstattungskataloge.

Die in diesem Beitrag diskutierten Ansätze zur Weiterentwicklung beziehen sich sowohl auf die Elemente von

Prof. Dr.-Ing. S. Greiving $(\bowtie) \cdot$ F. Flex

Institut für Raumplanung, Fakultät Raumplanung, TU Dortmund,

August-Schmidt-Str. 10,

44227 Dortmund, Deutschland

E-Mail: stefan.greiving@tu-dortmund.de

F. Flex

E-Mail: florian.flex@tu-dortmund.de

\section{Dr. T. Terfrüchte}

Fachgebiet Raumordnung und Planungstheorie,

Fakultät Raumplanung, TU Dortmund,

August-Schmidt-Str. 6,

44227 Dortmund, Deutschland

E-Mail: thomas.terfruechte@tu-dortmund.de
Zentrale-Orte-Konzepten als auch den Fortschreibungsprozess eines Landesraumordnungsplanes oder -programms. Für wesentlich wird erachtet, dass sich begründbare Einstufungen im Zentrale-Orte-Konzept auf eine empirische Überprüfung des Zentrale-Orte-Systems zurückführen lassen müssen. Für diese ist wiederum ein politisch legitimiertes Zielsystem erforderlich, aus dem sich Kriterien für die empirische Überprüfung und Einstufung von Zentralen Orten ableiten lassen. Dabei sollte der Plangeber die verwendete Methodik offenlegen.

Eine vorhandene Ausstattung mit zentralen Einrichtungen ist nicht hinreichend für eine Festlegung eines Zentralen Ortes. Dieser sollte vielmehr zudem bereichsbildend wirken und raumordnerisch erforderlich sein, um eine Versorgung mit Gütern und Dienstleistungen innerhalb einer zu definierenden zumutbaren Erreichbarkeit auch unter prognostischen Gesichtspunkten gewährleisten zu können. Deshalb ist auch eine raumstrukturelle Differenzierung erforderlich. Ausstattungsgleiche Orte in unterschiedlichen Raumstrukturen sollten deshalb ungleich behandelt werden können.

Schlüsselwörter Landesplanung · Zentraler Ort · Zentrale-Orte-Konzept · Validierung

\section{Comparative Study on the Central Place Concepts of the German Länder and Recommendations for their Further Development}

Abstract Central place concepts are an inherent part of all regional plans of the German federal states (the 'Län$d e r$ '). These concepts have become increasingly important because regional plans need to be adapted to recent and future demographic changes in order to ensure an adequate 
provision of services of general interest. This paper analyses the current central place concepts of the Länder and gives recommendations for their further development.

The analysis shows that the Länder have developed different approaches in regard to central place concepts, both in terms of definitions, threshold levels of population and accessibility and also their function for guiding future regional development.

The paper gives recommendations on how to improve central place concepts and related plan approval procedures. Policy makers and planning authorities should define and seek political approval for overarching goals, from which key analytical indicators would be derived. These indicators should then be used for an empirical analysis, which would finally allow designating central places and their respective functions. Of course, the underlying methodology should be explained in detail within each regional plan.

A central place should not be designated on the basis of its current facilities and services, but on the basis of the specific demographic and spatial characteristics and requirements of the surrounding region. Thus two cities with similar central facilities could nevertheless be handled differently by a central place concept because their respective hinterlands have different needs for central places.

Keywords Regional planning · Central places .

Central place concepts $\cdot$ Validation

\section{Einleitung}

Zentrale-Orte-Konzepte sind Bestandteil aller Pläne und Programme der Landes- und Regionalplanung der Flächenländer. Auch im 2008 neu gefassten Raumordnungsgesetz (ROG) wird in den Grundsätzen die Konzentration der Siedlungsentwicklung ( $§ 2$ Abs. 2 Nr. 2 Satz 2 ROG) und die Bündelung der sozialen Infrastruktur ( $§ 2$ Abs. 2 Nr. 3 Satz 2 ROG) auf die Zentralen Orte normiert.

Gerade im Zusammenhang mit den Herausforderungen, die aus dem demographischen Wandel für die raumordnerische Steuerung der Daseinsvorsorge erwachsen, wird den Zentrale-Orte-Konzepten eine zunehmende Bedeutung beigemessen (BMVBS 2010; Spannowsky 2011; Bartholomae/ Schoenberg 2013). Im Eckpunktebeschluss der Ministerkonferenz für Raumordnung (MKRO) zum Thema „Demographischer Wandel und Daseinsvorsorge" vom 29. April 2008 wird eine situationsadäquate „Handhabung des Zentrale-Orte-Konzepts" gefordert (MKRO 2008, S. 1). Dabei besteht ein Anpassungsdruck gerade in Schrumpfungsregionen - sowohl im Hinblick auf die Anzahl der Zentralen Orte als auch in Bezug auf ihre Ausweisungskriterien und ihre Steuerungswirkungen. Zugleich haben jüngere ober- gerichtliche Urteile in verschiedenen Bundesländern die Inkonsistenzen bestehender Zentrale-Orte-Konzepte und hier besonders die mangelnde Begründung bzw. Begründbarkeit der Einstufungen Zentraler Orte und ihrer Versorgungsbereiche aufgedeckt. Einen hohen Stellenwert wird den Zentrale-Orte-Konzepten auch in den „Leitbildern und Handlungsstrategien für die Raumentwicklung in Deutschland" (MKRO 2006) eingeräumt, insbesondere bei der Sicherung der Daseinsvorsorge (Leitbild 2). Daran wird im Entwurf der neuen Leitbilder im modifizierten Leitbild 2 auch festgehalten (MKRO 2013).

Das (eine) Zentrale-Orte-Konzept gibt es allerdings nicht (mehr). Vielmehr haben sich aus dem durch die MKRO 1968 (ergänzt 1972 und 1983) bundeseinheitlich definierten Rahmen unterschiedliche Steuerungskonzepte in den Ländern entwickelt. Damit einhergegangen ist auch ein unterschiedliches Verständnis hinsichtlich der elementaren Begriffe im Zusammenhang mit Zentralen Orten.

Wesentliche Ziele des Beitrags sind vor diesem Hintergrund die Darstellung und Analyse des Status quo der Zentrale-Orte-Konzepte in den Ländern sowie Empfehlungen zu ihrer Weiterentwicklung. ${ }^{1}$ Zunächst wird das Ergebnis einer umfassenden Bestandsaufnahme aller bestehenden Zentrale-Orte-Konzepte in den Flächenländern vorgestellt (Kap. 2, Stichtag 30.06.2014); diese geht auf eine Dokumentenanalyse (geltende Landesraumordnungspläne und -programme) und ergänzende Interviews mit Vertretern der Landesplanungsbehörden zurück. Im Anschluss werden an den Befund anknüpfend und unter Würdigung der identifizierten Defizite sowohl inhaltliche Empfehlungen zur Weiterentwicklung und Stärkung (Kap. 3) wie auch prozedurale Empfehlungen zur Aufstellung bzw. Fortschreibung (Kap. 4) von Zentrale-Orte-Konzepten beleuchtet. Der Beitrag endet mit einem Fazit und Anknüpfungspunkten für weitere Forschungsarbeiten (Kap. 5).

\section{Zentrale-Orte-Konzepte in den Ländern}

Bei aller Unterschiedlichkeit der Zentrale-Orte-Konzepte in den Flächenländern (die Stadtstaaten Bremen und Hamburg sind aufgrund fehlender Landesraumordnungspläne nicht berücksichtigt) lassen sich dennoch Gemeinsamkeiten unter den Ländern in der Anlage erkennen: Neben den Grundmerkmalen (Hierarchiestufen und Mehrfachorte) sind dies Ausweisungskriterien, Ausstattungskataloge und Aussagen

\footnotetext{
${ }^{1}$ Die in diesem Beitrag präsentierten Ergebnisse basieren im Wesentlichen auf dem Ressortforschungsprojekt „Reform der Zentrale-Orte-Konzepte in den Ländern und Folgen für Siedlungsstruktur und Daseinsvorsorge", das die Verfasser im Auftrag des Bundesministeriums für Verkehr und digitale Infrastruktur (BMVI) und des Bundesinstituts für Bau-, Stadt- und Raumforschung (BBSR) durchgeführt haben.
} 
Tab. 1 Anzahl Zentraler Orte nach Hierarchiestufen. (Eigene Darstellung auf Grundlage der geltenden Pläne und Programme der Länder (siehe Rechtsquellenverzeichnis))

\begin{tabular}{llllllll}
\hline & \multicolumn{7}{l}{ Anzahl Zentraler Orte nach Hierarchiestufen } \\
\cline { 2 - 8 } & K1Z & UZ & GZ & MZ & OZ & M & Teilfunktionen \\
\hline Baden-Württemberg & 196 & 111 & - & 85 & 14 & - & MZTFOZ (1) \\
Bayern & - & - & 657 & 156 & 30 & - & - \\
Berlin-Brandenburg & - & - & - & 42 & 4 & 1 & - \\
Hessen $^{\mathrm{a}}$ & 80 & 44 & 191 & 91 & 10 & - & MZTFOZ (4) \\
Mecklenburg-Vorpommern & - & - & 75 & 18 & 4 & - & - \\
Niedersachsen & - & - & 313 & 78 & 11 & - & MZTFOZ (6), GZTFMZ (11) \\
Nordrhein-Westfalen & - & - & 188 & 192 & 16 & - & Möglich, aber nicht ausgewiesen \\
Rheinland-Pfalz & - & - & 150 & 83 & 5 & - & MZTFOZ (5), metropolitane TF (2) \\
Saarland & - & - & 40 & 11 & 1 & - & - \\
Sachsen & - & - & 80 & 38 & 6 & - & - \\
Sachsen-Anhalt & - & - & 102 & 20 & 3 & - & MZTFOZ (2), GZTFMZ (5) \\
Schleswig-Holstein & - & 35 & - & 13 & 4 & - & UZTFMZ (9), Stadtrandkern I. Ordnung TFMZ ${ }^{\mathrm{b}}$ (0) \\
Thüringen & - & - & 76 & 24 & 3 & - & MZTFOZ (8) \\
\hline
\end{tabular}

KlZ Kleinzentrum, $U Z$ Unterzentrum, $G Z$ Grundzentrum, $M Z$ Mittelzentrum, $O Z$ Oberzentrum, $M$ Metropole, $T F$ Teilfunktion,

MZTFOZ Mittelzentrum mit Teilfunktionen eines Oberzentrums, GZTFMZ Grundzentrum mit Teilfunktionen eines Mittelzentrums,

UZTFMZ Unterzentrum mit Teilfunktion eines Mittelzentrums

${ }^{a}$ In Hessen werden GZ in Nord- und Mittelhessen festgelegt, während in Südhessen weiterhin KIZ und UZ bestehen

${ }^{\mathrm{b} S t a n d r a n d k e r n e ~ I . ~ O r d n u n g ~ m i t ~ T e i l f u n k t i o n ~ e i n e s ~ M i t t e l z e n t r u m s ~ s t e h e n ~ a l s ~ G l i e d e r u n g s s t u f e ~ n o c h ~ z u r ~ V e r f u ̈ g u n g, ~ w u r d e n ~ a b e r ~ i m ~ a k t u e l l e n ~}$ Zentrale-Orte-Konzept nicht ausgewiesen

zu zentralörtlichen Versorgungsbereichen, wobei letztere mitunter die Ausweisungskriterien dominieren.

\subsection{Grundmerkmale von Zentrale-Orte-Konzepten: Hierarchiestufen und Mehrfachorte}

Die deutliche Mehrheit der Flächenländer bezieht den Zentralen Ort auf die politische Gemeinde als Normadressat. Die Landesplanungsbehörden sehen bei großen Flächengemeinden oder Gemeinden mit mehreren Ortsteilen jedoch die Notwendigkeit zur räumlichen Konzentration der zentralörtlichen Funktionen am funktionalen Hauptort vor (was dem theoretisch-analytischen Begriffsverständnis des Zentralen Orts als Standortcluster entspricht) (Tab. 1).

Hinsichtlich der Hierarchiestufen dominiert grundsätzlich ein dreistufiges Zentrale-Orte-Konzept aus Grund-, Mittel- und Oberzentren, wobei auf der grundzentralen Ebene in Baden-Württemberg und Teilen von Hessen zwischen Unter- und Kleinzentren differenziert wird. Die gemeinsame Landesplanung Berlin-Brandenburg verzichtet als einzige Landesplanung auf die Ausweisung Zentraler Orte der grundzentralen Ebene und weist als einzige eine Metropole im Zentrale-Orte-Konzept aus. Die mitunter gravierenden Schwankungen in der absoluten Anzahl ausgewiesener Orte je Stufe (bei den Grundzentren zwischen 75 in Mecklenburg-Vorpommern und 657 in Bayern oder einem Oberzentrum im Saarland und 30 in Bayern) sind vor allem auf die unterschiedlichen Gemeindegebietsstrukturen, die Größe der Bundesländer und die unterschiedlichen Ausweisungskriterien zurückzuführen.
Mit Ausnahme von Nordrhein-Westfalen und dem Saarland sind in allen Flächenländern Mehrfachorte ausgewiesen, wobei eine große Heterogenität der verwendeten Begriffe zu konstatieren ist (vgl. Tab. 2).

Zugleich zeigt sich, dass mit den festgelegten Mehrfachorten ganz unterschiedliche Erwartungshaltungen seitens der Landesplanung an die Funktionsempfänger einhergehen, die durch unterschiedlich strikte (Kooperations-) Anforderungen an die Teilorte zum Ausdruck kommen; in vier der 11 Flächenländer mit ausgewiesenen Mehrfachorten sind keine derartigen Anforderungen formuliert, so dass auch nicht von Funktionsteilungen gesprochen werden kann.

\subsection{Ausweisungskriterien für Zentrale Orte}

Die Festlegungen zu Ausweisungskriterien unterscheiden sich mitunter deutlich zwischen den Ländern: Die Konkretisierung reicht je nach Zentralitätsstufe von Verweisen auf MKRO-Entschließungen über dezidierte Einwohnerschwellenwerte und dem Kriterium der Arbeitsplatzzentralität (die Zahl der Einpendler ist größer als die Zahl der Auspendler) bis hin zu differenzierten Anforderungen an die Entwicklungsfähigkeit des Zentralen Ortes. Die Bindungswirkung reicht - teils getrennt nach Hierarchiestufen - von Begründungen über Soll-Bestimmungen in den Grundsätzen bis hin zu verbindlichen Zielen. Die Legitimation der Ausweisungskriterien ist schließlich teilweise direkt (insbesondere Erreichbarkeits- und Tragfähigkeitskriterien), teilweise 
Tab. 2 Mehrfachorte und Kooperationsanforderungen. (Eigene Darstellung auf Grundlage der geltenden Pläne und Programme der Länder (siehe Rechtsquellenverzeichnis))

\begin{tabular}{|c|c|c|c|c|}
\hline & \multicolumn{4}{|c|}{ Mehrfachorte und Kooperationsanforderungen } \\
\hline & Mehrfachorte & Kooperationsanforderungen & Verwendeter Begriff & Anzahl (GZ/MZ/OZ) \\
\hline Baden-Württemberg & $\mathrm{Ja}$ & Nein & „Doppel- und Mehrfachzentrum“ & $29 / 9 / 5$ \\
\hline Bayern & $\mathrm{Ja}$ & $\mathrm{Ja}$ & „Zentraler Doppel- und Mehrfachort“ & $-/ 26 / 4$ \\
\hline Berlin-Brandenburg & $\mathrm{Ja}$ & $\mathrm{Ja}$ & „Mittelzentrum in Funktionsteilung“ & $-/ 8 /-$ \\
\hline Hessen & $\mathrm{Ja}$ & Nein & $\begin{array}{l}\text { „Zentraler Ort in gemeinsamer } \\
\text { Funktionsteilung“ }\end{array}$ & $-/ 3 / 1$ \\
\hline Mecklenburg-Vorpommern & $\mathrm{Ja}$ & $\mathrm{Ja}$ & „Gemeinsame Zentrale Orte“ & $2 /-/ 1$ \\
\hline Niedersachsen & $\mathrm{Ja}$ & Nein & „Zentrenverbund“ & $-/ 1 / 1$ \\
\hline Nordrhein-Westfalen & Nein & - & - & $-1-1-$ \\
\hline Rheinland-Pfalz & $\mathrm{Ja}$ & $\mathrm{Ja}$ & $\begin{array}{l}\text { „Zentraler Verbund kooperierender } \\
\text { Zentren“ }\end{array}$ & $-/ 11 /-$ \\
\hline Saarland & Nein & - & - & $-1-1-$ \\
\hline Sachsen & $\mathrm{Ja}$ & $\mathrm{Ja}$ & „Zentralörtlicher Städteverbund““ & $11 / 3 / 1$ \\
\hline Sachsen-Anhalt & $\mathrm{Ja}$ & $\mathrm{Ja}$ & Teilung zentralörtlicher Aufgaben & $4 /-1-$ \\
\hline Schleswig-Holstein & $\mathrm{Ja}$ & Nein & Gemeinsame Funktionswahrnehmung & $4 / 2 /-$ \\
\hline Thüringen & $\mathrm{Ja}$ & $\mathrm{Ja}$ & „Funktionsteilige Zentrale Orte“ & $5 / 5 /-$ \\
\hline
\end{tabular}

indirekt (z. B. positives Pendlersaldo) auf die Regelungen im ROG zurückzuführen.

Vier der 13 Flächenländer haben keine konkreten Ausweisungskriterien für die Zentralen Orte der verschiedenen Hierarchiestufen in den Plänen und Programmen verankert (Tab. 3). Teilweise werden auch allgemeine Kriterien aufgeführt, z. B. die Berücksichtigung siedlungsstruktureller Gegebenheiten in Baden-Württemberg oder der Verweis auf MKRO-Entschließungen in Hessen und im Saarland.

Die übrigen Länder verweisen i. d. R. auf Einwohnerschwellenwerte (im Sinne von Tragfähigkeitskriterien) für die Zentralen Orte selbst (städtischer Siedlungskern und/ oder Gemeinde) und ihre zugeordneten zentralörtlichen Bereiche sowie allgemeine Erreichbarkeitserfordernisse als Ausweisungskriterien. Darüber hinaus werden häufig folgende Kriterien für die Einstufung herangezogen, die auch die Entwicklungsfunktion Zentraler Orte berücksichtigen: Funktion als Verkehrsknoten, Arbeitsplatzangebot bzw. die Anzahl sozialversicherungspflichtig Beschäftigter und Einpendler. In Einzelfällen werden auch pauschale Regelungen zur Einstufung getroffen, wie etwa im Saarland, wo all jene Gemeindehauptorte als Grundzentren ausgewiesen werden, die weder Mittel- noch Oberzentrum sind. Insbesondere hinsichtlich der Schwellenwerte für Einwohner, Beschäftigte und Einpendler bestehen jedoch mitunter große Unterschiede (Tab. 3) erstens hinsichtlich der Streuung und zweitens hinsichtlich der Differenzierung, die i. d. R. nach Raumkategorien erfolgt.

\subsection{Ausstattungskataloge}

Ausgenommen Nordrhein-Westfalen haben sämtliche Flächenländer zentralörtliche Ausstattungskataloge in ihre Pläne und Programme aufgenommen. Bis auf das Saar- land, wo keine Aussagen zur grundzentralen Stufe getroffen werden, beziehen sich diese Ausstattungskataloge auf jede der landesweit festgelegten Stufen. Hinsichtlich der Bindungswirkung weisen die Plangeber i. d. R. auf die Orientierungsfunktion bzw. den beispielhaften Charakter der Ausstattungskataloge hin. Einzige Ausnahme ist hier Rheinland-Pfalz, wo im LEP IV zwischen , anzustrebender verbindlicher Ausstattung“ und „notwendiger Ausstattung im Regelfall“" unterschieden wird; grundsätzlich hat der Katalog auch dort Orientierungsfunktion.

14 Funktionsbereiche lassen sich in den 12 Landesraumordnungsplänen identifizieren (Tab. 4): Während Ausstattungsmerkmale aus den Bereichen Bildung und Gesundheit in allen 12 Plänen mindestens einer Hierarchiestufe zugeordnet sind, gibt es zudem häufige Nennungen (in mindestens 10 der 12 Pläne) in den Bereichen Einzelhandel, Kultur, Öffentliche Verwaltung, Sport und Verkehr. Weniger häufig werden Ausstattungen in den Bereichen Arbeitsmarkt, Gefahrenabwehr, Soziales sowie Wissenschaft und Forschung genannt. Kleinere Unterschiede gibt es bei der Zuordnung einzelner Einrichtungen zu den Hierarchiestufen, so werden etwa Schwerpunktkrankenhäuser teils als oberzentral und teils als mittelzentral eingestuft oder wissenschaftlich geführte Bibliotheken oder Fachärzte teils als mittelzentral und teils als grundzentral. Sowohl die Funktionsbereiche insgesamt (ausgenommen die auch in den Plänen und Programmen weniger häufig genannten Bereiche Soziales und Gefahrenabwehr) als auch die Ausstattungsmerkmale decken sich im Wesentlichen mit den EntschlieBungen der MKRO aus den Jahren 1968, 1972 und 1983 (vgl. hierzu auch Terfrüchte 2015, S. 98 ff.). 
Tab. 3 Ausweisungskriterien für Zentrale Orte. (Eigene Darstellung auf Grundlage der geltenden Pläne und Programme der Länder (siehe Rechtsquellenverzeichnis))

\begin{tabular}{|c|c|c|c|}
\hline & \multicolumn{3}{|l|}{ Ausweisungskriterien für Zentrale Orte } \\
\hline & Grundzentral & Mittelzentral & Oberzentral \\
\hline Baden-Württemberg & - & - & - \\
\hline Bayern & $\begin{array}{l}\text { Ja } \\
\text { (Mitversorgung, grundzentrale } \\
\text { Einrichtungen) }\end{array}$ & $\begin{array}{l}\text { Ja } \\
\text { (Standortvoraussetzungen, } \\
\text { Erreichbarkeit) }\end{array}$ & $\begin{array}{l}\text { Ja } \\
\text { (Standortvoraussetzungen, } \\
\text { Erreichbarkeit) }\end{array}$ \\
\hline Berlin-Brandenburg & - & $\begin{array}{l}\text { Ja } \\
\text { (Standortvoraussetzungen, } \\
\text { Erreichbarkeit) }\end{array}$ & $\begin{array}{l}\text { Ja } \\
\text { (Stellenwert im Städtesystem, Lage) }\end{array}$ \\
\hline Hessen & $\begin{array}{l}\text { UZ: Ja } \\
\text { (3000 EW im städtischen Kern, Mitver- } \\
\text { sorgung, grundzentrale Einrichtungen) }\end{array}$ & $\begin{array}{l}\mathrm{Ja} \\
\text { ( } 7000 \mathrm{EW} \text { im ZO, mittelzentrale } \\
\text { Einrichtungen, Nahverkehrsknoten) }\end{array}$ & $\begin{array}{l}\text { Ja } \\
(100.000 \text { EW im städtischen } \\
\text { Kern, oberzentrale Einrichtungen, } \\
\text { Regionalverkehrsknoten) }\end{array}$ \\
\hline $\begin{array}{l}\text { Mecklenburg-Vorpom- } \\
\text { mern }\end{array}$ & $\begin{array}{l}\text { Ja } \\
(2000 \text { EW (LR) bzw. } 5000 \text { EW (SUR)/ } \\
\text { Siedlungskern, } 5000 \text { EW/Bereich, } 600 \\
\text { Beschäftigte, } 300 \text { Einpendler, grundzent- } \\
\text { rale Einrichtungen) }\end{array}$ & $\begin{array}{l}\text { Ja } \\
(10.000 \mathrm{EW} / \mathrm{Gemeinde}, 30.000 \mathrm{EW} / \\
\text { Bereich, } 4000 \text { Beschäftigte, } 2000 \\
\text { Einpendler) }\end{array}$ & $\begin{array}{l}\text { Ja } \\
\text { (70.000 EW/Gemeinde, } 300.000 \\
\text { EW/Bereich, } 30.000 \text { Beschäftigte, } \\
\text { 15.000 Einpendler) }\end{array}$ \\
\hline Niedersachsen & - & $\begin{array}{l}\text { Ja } \\
(20.000 \text { EW/Gemeinde, } 35.000 \\
\text { EW/Bereich, } 10.000 \text { Beschäftigte, } \\
\text { 4000 Einpendler, mittelzentrale } \\
\text { Einrichtungen) }\end{array}$ & $\begin{array}{l}\text { Ja } \\
\text { (60.000 EW/Gemeinde, } 300.000 \\
\text { EW/Bereich, 25.000 Beschäftig- } \\
\text { te, } 9000 \text { Einpendler, oberzentrale } \\
\text { Einrichtungen) }\end{array}$ \\
\hline Nordrhein-Westfalen & - & - & - \\
\hline Rheinland-Pfalz & - & - & - \\
\hline Saarland & - & - & - \\
\hline Sachsen & $\begin{array}{l}\text { Ja } \\
\text { (Tragfähigkeit, ÖPNV-Knoten, grund- } \\
\text { zentrale Einrichtungen) }\end{array}$ & $\begin{array}{l}\text { Ja } \\
\text { (10.000 EW (LR) bzw. 15.000 EW/ } \\
\text { Gemeinde, } 3000 \text { Beschäftigte (LR) } \\
\text { bzw. } 5000 \text { Beschäftigte, } 45.000 \mathrm{EW/} \\
\text { Bereich) }\end{array}$ & $\begin{array}{l}\text { Ja } \\
(50.000 \text { EW/Gemeinde, Lage, über- } \\
\text { regionaler Verkehrsknoten, } 20.000 \\
\text { Beschäftigte, } 10.000 \text { Einpendler, } \\
\text { Erreichbarkeit) }\end{array}$ \\
\hline Sachsen-Anhalt & $\begin{array}{l}\mathrm{Ja} \\
(3000 \mathrm{EW} / \mathrm{ZO}, 12.000 \mathrm{EW} / \mathrm{Be}- \\
\text { reich, grundzentrale Versorgung, } \\
\text { Erreichbarkeit) }\end{array}$ & $\begin{array}{l}\text { Ja } \\
(20.000 \mathrm{EW} / \text { Gemeinde, } 70.000 \mathrm{EW} / \\
\text { Bereich, mittelzentrale Versorgung, } \\
\text { Erreichbarkeit) }\end{array}$ & $\begin{array}{l}\text { Ja } \\
\text { (300.000 EW/Bereich, oberzentrale } \\
\text { Versorgung, Arbeitsmarktzentrum, } \\
\text { Erreichbarkeit) }\end{array}$ \\
\hline Schleswig-Holstein ${ }^{\mathrm{a}}$ & $\begin{array}{l}\text { UZ: Ja } \\
4000 \mathrm{EW} / \mathrm{Gemeinde}, 10.000 \mathrm{EW} / \\
\text { Bereich } \\
\text { (3500 bzw. } 7000 \text { in strukturschwachen } \\
\text { ländlichen Räumen) }\end{array}$ & $\begin{array}{l}\text { Ja } \\
\text { (15.000 EW bzw. } 25.000 \text { EW (VR)/ } \\
\text { Gemeinde, } 40.000 \text { EW bzw. } 80.000 \\
\text { EW (VR)/Bereich) }\end{array}$ & - \\
\hline Thüringen & $\begin{array}{l}\text { Ja } \\
\text { (5000 EW/Bereich mit Demographiefak- } \\
\text { tor, Funktionserfüllung, Erreichbarkeit) }\end{array}$ & $\begin{array}{l}\text { Ja } \\
\text { (Funktionserfüllung, Erreichbarkeit, } \\
\text { Einzugsbereich) }\end{array}$ & $\begin{array}{l}\text { Ja } \\
\text { (i. d. R. } 100.000 \mathrm{EW} / \text { Gemeinde, } \\
\text { Funktionserfüllung) }\end{array}$ \\
\hline
\end{tabular}
EW Einwohner, $L R$ ländlicher Raum, VR Verdichtungsraum, SUR Stadt-Umland-Raum, UZ Unterzentrum, ZO Zentraler Ort

${ }^{a}$ Die Stadtrandkerne in Schleswig-Holstein sollen „baulich so geordnet und gestaltet sein, dass ein Versorgungskern erkennbar ist“ (LEP S-H 2010, G 2.2.5.2)

\subsection{Zentralörtliche Versorgungsbereiche}

In 11 Flächenländern werden zentralörtliche Bereiche festgelegt, wenn auch nicht immer für jede Stufe. Begrifflich bestehen insofern Unterschiede, als einzelne Länder vom (normativen) Versorgungsbereich, andere vom (deskriptiven) Verflechtungsbereich und wieder andere vom (aus der Einzelhandelssteuerung bekannten) Einzugsbereich sprechen. Begriffliche Übereinstimmung besteht jedoch bei der Bezeichnung der Bereiche auf den drei Hierarchiestufen:
Nahbereiche - auf der grundzentralen Ebene - werden in sieben Flächenländern ausgewiesen, Mittelbereiche in neun und Oberbereiche in zwei Flächenländern. Darüber hinaus werden in einigen Ländern zentralörtliche Bereiche zwar erwähnt, allerdings weder textlich noch zeichnerisch dargestellt (z. B. Nordrhein-Westfalen). Teilweise wird explizit auf den (reinen) Analysezweck der Abgrenzung verwiesen: z. B. Nahbereiche in Baden-Württemberg (allgemeine Analyse) oder Mittelbereiche in Rheinland-Pfalz (Grundlage für den Finanzausgleich). 
Tab. 4 Funktionsbereiche in den Ausstattungskatalogen. (Eigene Darstellung auf Grundlage der geltenden Pläne und Programme der Länder (siehe Rechtsquellenverzeichnis))

\begin{tabular}{lllll}
\hline & \multicolumn{4}{l}{ Funktionsbereiche in den } \\
& \multicolumn{4}{l}{ Ausstattungskatalogen } \\
\cline { 2 - 5 } & zentral & $\begin{array}{l}\text { Mittel- } \\
\text { zentral }\end{array}$ & $\begin{array}{l}\text { Ober- } \\
\text { zentral }\end{array}$ & $\begin{array}{l}\text { Ge- } \\
\text { samt }^{\mathrm{a}}\end{array}$ \\
\hline Bildung & 8 & 11 & 12 & 12 \\
Gesundheit & 9 & 11 & 10 & 12 \\
Einzelhandel & 8 & 6 & 8 & 11 \\
Öffentliche Verwaltung & 5 & 8 & 9 & 11 \\
Kultur & 6 & 9 & 10 & 10 \\
Sport & 7 & 9 & 8 & 10 \\
Verkehr & 8 & 8 & 8 & 10 \\
Finanz- und & 7 & 3 & 4 & 9 \\
Versicherungswesen & & & & \\
Sonstige Dienstleistungen & 7 & 2 & 2 & 8 \\
Rechtspflege & - & 8 & 7 & 8 \\
Soziales & 3 & 6 & 2 & 6 \\
Wissenschaft und & - & 1 & 6 & 6 \\
Forschung & & & & \\
Gefahrenabwehr & 3 & 3 & 1 & 5 \\
Arbeitsmarkt & 1 & 2 & 1 & 3
\end{tabular}

${ }^{a}$ Die Angabe in dieser Spalte bezieht sich auf die Anzahl der Nennungen des jeweiligen Funktionsbereichs in den Plänen aller Länder insgesamt, nicht auf die Summen aus den einzelnen Hierarchiestufen

In Tab. 5 ist dargestellt, nach welchen Kriterien die zentralörtlichen Bereiche abgegrenzt sind. Dabei ist zu unterscheiden zwischen allgemeinen Abgrenzungskriterien, etwa dass die Abgrenzung anhand sozioökonomischer Verflechtungen erfolgt, und konkreten methodischen Hinweisen. Dazu zählen etwa Zuordnungsregeln wie etwa Schwellenwerte für Einfach- oder Mehrfachzuordnung oder Prämissen, z. B. dass Mittelbereiche sich aus Nahbereichen zusammensetzen oder keine Kreisgrenzen zerschneiden sollen (Verwaltungskongruenz).

Hinsichtlich der Abgrenzungskriterien überwiegt die Berücksichtigung von Erreichbarkeits- und Tragfähigkeitskriterien sowie der Pendlerverflechtung, die teilweise stellvertretend für (multifunktionale) sozioökonomische Verflechtungen herangezogen werden. Bei den methodischen Hinweisen wird im Wesentlichen auf die Gewährleistung der Verwaltungskongruenz verwiesen, also eine unzerteilte Zuordnung von politischen Gemeinden (zu Nah- und Mittelbereichen) und Kreisen (zu Oberbereichen) bzw. die Binnengliederung von Kreisen in Mittel- und Nahbereiche.

Differenziert nach Hierarchiestufen zeigt sich folgendes Bild: Bei den Nahbereichen liegt der Schwerpunkt auf der überwiegenden Orientierung der Bevölkerung, wobei dies nicht weiter operationalisiert wird; teilweise wird hier vom Prinzip der Ausrichtung nach räumlicher Nähe ausgegangen. Mitunter wird - sofern Zentrale Orte nicht mit dem Gemeindegebiet gleichgesetzt sind - auch pauschal festge-
Tab. 5 Abgrenzung zentralörtlicher Bereiche. (Eigene Darstellung auf Grundlage der geltenden Pläne und Programme der Länder (siehe Rechtsquellenverzeichnis))

\begin{tabular}{lll}
\hline & \multicolumn{2}{l}{ Abgrenzung zentralörtlicher Bereiche } \\
\cline { 2 - 3 } & $\begin{array}{l}\text { Allgemeine } \\
\text { Abgrenzungskriterien }\end{array}$ & $\begin{array}{l}\text { Methodische } \\
\text { Hinweise }\end{array}$ \\
\hline Baden-Württemberg & $\begin{array}{l}\text { Orientierung, Erreichbarkeit, } \\
\text { Tragfähigkeit }\end{array}$ & - \\
Bayern & Orientierung & - \\
Berlin-Brandenburg & $\begin{array}{l}\text { Raumstrukturelle Zusam- } \\
\text { menhänge, Erreichbarkeit }\end{array}$ & Verwaltungs- \\
& kongruenz \\
Hessen & Erreichbarkeit & - \\
Mecklenburg-Vor- & Sozioökonomische Ver- & Verwaltungs- \\
pommern & flechtungen, Berufs- und & kongruenz \\
& Versorgungspendlerströme & \\
Niedersachsen & Raumstrukturelle Zusam- & - \\
& menhänge (z. B. Entfernung) & \\
Nordrhein-Westfalen & - & - \\
Rheinland-Pfalz & Erreichbarkeit & - \\
Saarland & Erreichbarkeit, sozioökono- & - \\
& mische Verflechtungen & \\
Sachsen & Sozioökonomische & - \\
& Verflechtungen, insb. & \\
Sachsen-Anhalt & Pendlerverflechtungen & - \\
Schleswig-Holstein & Erreichbarkeit & - \\
Thüringen & Orientierung & - \\
& Erreichbarkeit, & Pendlerausrichtung \\
\hline
\end{tabular}

legt, dass die jeweiligen Gemeindegebiete den Nahbereich der Zentralen Orte bilden. Bei den Mittelbereichen überwiegen i. d. R. Erreichbarkeits- und Tragfähigkeitskriterien.

In vielen Ländern wird der Mehrfachausrichtung der Bevölkerung hinsichtlich ihrer Versorgungsbeziehungen explizit - wenn auch mit unterschiedlichem Ergebnis Rechnung getragen: Sachsen weist z. B. auf die „Unmöglichkeit" einer eindeutigen Abgrenzung von Oberbereichen hin und verzichtet dementsprechend auf eine Darstellung; bei den Mittelbereichen hingegen sind räumliche Überschneidungen explizit vorgesehen. In Berlin-Brandenburg sind räumliche Überlappungen grundsätzlich ausgeschlossen, während in Bayern gemeinsame Nahbereiche mehrerer Grundzentren möglich sind; im Saarland ist pauschal das gesamte Bundesland als Oberbereich der Landeshauptstadt Saarbrücken ausgewiesen.

\section{Inhaltliche Empfehlungen zur Weiterentwicklung und Stärkung der Zentrale-Orte-Konzepte}

Auf der Grundlage der in Kap. 2 vorgestellten Analyse der bestehenden Pläne und Programme der Länder erfolgt nun eine Diskussion von Ansatzpunkten für eine Weiterentwicklung der Zentrale-Orte-Konzepte. Dabei gilt es einerseits, ein gemeinsames Begriffsverständnis und methodische Erfordernisse herauszustellen, die für alle Länder gelten 
sollten, und andererseits die Unterschiedlichkeit der Bundesländer und ihrer Regionen ebenso zu berücksichtigen wie die Aufgabenverantwortung der Länder für die Landesraumordnung. Auf die Bedeutung der zentralörtlichen Festlegungen für verschiedene Themenbereiche wie Siedlungsflächenentwicklung, Sicherung der Daseinsvorsorge, großflächiger Einzelhandel, gewerbliche Wirtschaft oder den kommunalen Finanzausgleich wird dabei bewusst nicht näher eingegangen, weil dies den Rahmen des Aufsatzes sprengen würde. Zudem hängt die konkrete Steuerungswirkung auch von verschiedenen, länderspezifischen Faktoren wie etwa der jeweiligen institutionellen Verfasstheit der Raumordnung ab.

\subsection{Hierarchiestufen und Mehrfachorte}

In der raumordnerischen Praxis werden meist politische Gemeinden als Zentrale Orte ausgewiesen (Territorialprinzip) und nicht der Standortcluster als eigentlicher Zentraler Ort. Ein wesentlicher Vorteil einer am Standortcluster orientierten Ausweisungspolitik besteht zweifellos in der feinteiligen Steuerungswirkung der Zentrale-Orte-Konzepte und damit einer effektiven Zielerreichung hinsichtlich des Konzentrations- und Bündelungsprinzips. Gleichwohl gibt es auch Argumente für das Territorialprinzip, die insbesondere auf die Verwaltungsfunktion (territoriale Zuständigkeit von Verwaltungseinrichtungen) als einem Faktor in Zentrale-Orte-Konzepten abzielen. Die Bedeutung einer politisch selbstständigen Einheitsgemeinde für die Daseinsvorsorge wird auf diese Weise ebenso reflektiert wie die Tatsache, dass sich viele Infrastrukturen in Trägerschaft der Kommunen (Gemeinden bzw. Landkreisen) befinden, die somit Adressaten der Funktionszuweisung innerhalb des ZentraleOrte-Konzepts sind. Aus diesem Grund kann eine gebietsscharfe Festlegung Zentraler Orte (etwa über den Hauptort) auch als Einstufungskriterium für politische Gemeinden (als Normadressaten) verstanden werden, um die beabsichtigte Konzentration und Bündelung zentralörtlich bedeutsamer Infrastrukturen auch in großen Gemeinden mit vielen Ortsteilen sicherzustellen.

Dabei ist insbesondere auf grundzentraler Ebene ein räumlich gebündeltes Angebot an öffentlichen und privaten Dienstleistungs- und Versorgungseinrichtungen vorzusehen, um eine räumliche Kongruenz zwischen Standortcluster und normativer Steuerung zu erreichen. Eine Differenzierung in Klein- und Unterzentren - wie sie ohnehin nur in Baden-Württemberg und Teilen Hessens erfolgt - ist nur denkbar, wenn diese auch in einem eigenen Versorgungsbereich Versorgungsaufgaben für Dritte übernehmen, eine Differenzierung in einen zweistufigen Nahbereich erscheint hingegen wenig zweckdienlich und wäre kaum praktikabel (Blotevogel 2002, S. XXVI). Insofern wird empfohlen, auf der grundzentralen Ebene ausschließlich Grundzentren mit den zugehörigen Nahbereichen auszuweisen. Dies schließt Funktionsteilungen auf grundzentraler Ebene nicht aus, solange sichergestellt ist, dass tatsächlich eine Funktionsergänzung zwischen interagierenden Teilzentren besteht (Bartsch 2006; Greiving 2006; BMVBS 2008).

Bei Mittel- und Oberzentren erscheint - jenseits ihrer grundzentralen Cluster - eine Beschränkung der mit dem Zentrale-Orte-Konzept zu steuernden Einrichtungen auf räumliche Cluster nicht zwingend geboten, zumal viele zentralörtlich relevante Einrichtungen (z. B. Standorte weiterführender Schulen, Sportstätten, Tagungs- und Messeeinrichtungen, Häfen und Flughäfen) häufig auch außerhalb der Hauptorte vorliegen. Sind solche Einrichtungen Gegenstand der Ausweisungskriterien, blieben sie bei einer Orientierung an Standortclustern häufig unberücksichtigt. Hinsichtlich der normativen Steuerungswirkung ist es in Anlehnung an die Praxis in Rheinland-Pfalz denkbar, zu unterscheiden zwischen Einrichtungen, die im Mittelzentrum lokalisiert sein sollten, und jenen, die auch aus dem Mittelbereich heraus zur Versorgung der Bereichsbevölkerung beitragen können. Insofern sollte auch von der Ausweisung Zentraler Orte mit Teilfunktionen höherer Stufen abgesehen werden. Für derartige Zwischenstufen (z. B. Mittelzentren mit Teilfunktionen eines Oberzentrums) gilt das Gleiche wie für mehrere Stufen auf der grundzentralen Ebene: An eine solche Ausweisung müsste die Ausweisung zugeordneter Versorgungsbereiche der jeweiligen Zwischenstufe geknüpft sein, da andernfalls unklar wäre, für welche Dritten die Versorgung mit den Einrichtungen der höheren Stufe erfolgen soll. Zur Gewährleistung einer flächendeckenden Erreichbarkeit müsste alternativ die Ausweisung von Mehrfachorten geprüft werden, die dann gemeinsam das vollständige Funktionsspektrum der höheren Stufe für einen gemeinsamen Versorgungsbereich vorhalten.

\subsection{Ausweisungskriterien für Zentrale Orte}

Zunächst ist klarzustellen, dass eine Differenzierung der Ausweisungskriterien bzw. der Festlegung Zentraler Orte nach Raumstrukturen auf allen Hierarchiestufen erforderlich erscheint. Ziele der Raumordnung müssen im Sinne der Normklarheit für den Normadressaten räumlich und sachlich bestimmt bzw. zumindest bestimmbar sein (Urteil des BVerwG 4 C 8.10 vom 16.12.2010). Demnach erfüllen landesplanerische Vorschriften die Merkmale eines Ziels der Raumordnung, wenn die Voraussetzungen im Einzelfall im Wege der Auslegung eines Plans bestimmt oder wenigstens hinreichend bestimmbar sind (Bartram 2010, S. 47 f.).

Die räumliche und sachliche Bestimmbarkeit raumordnerischer Ziele ist aufgrund der mit den Zielfestlegungen verbundenen Eingriffe in das kommunale Selbstverwaltungsrecht zwingend erforderlich, um dem VerhältnismäBigkeitsprinzip und dem Willkürverbot Rechnung zu tragen. 
Dabei muss nachvollziehbar sein, welche Erwägungen bzw. überörtlichen Interessen von höherem Gewicht den Erlass der Rechtsnorm rechtfertigen. Diese Erwägungen unterscheiden sich zwischen den verschiedenen Raumstrukturen deutlich und bedürfen entsprechend maßgeschneiderter Ausweisungskriterien für Zentrale Orte und ihre Versorgungsbereiche.

Der VerfGH Münster (VerfGH 18/08 zum FOC Ochtrup, das heißt im Kontext der Steuerung des großflächigen Einzelhandels) argumentiert: „Mit Rücksicht darauf, dass die Verbotsregelung sich als erhebliche Beschränkung der Planungshoheit auswirkt, unterliegen die Schwellenwerte einem besonderen Rechtfertigungsbedürfnis." Der VerfGH hat auch das Erfordernis einer raumstrukturell differenzierten Betrachtung und hier explizit differenzierte Schwellenwerte für Zentrale Orte betont (VerfGH NRW 18/08, Rn. 23).

Mit wenigen Ausnahmen sind für Zentrale Orte und ihre Versorgungsbereiche Tragfähigkeits- und Erreichbarkeitsschwellen als Ausweisungskriterien festgelegt. Tragfähigkeitsschwellen - meist in Bezug auf die Wohnbevölkerung - sind jedoch nur Proxy-Indikatoren für die tatsächliche Tragfähigkeit zentraler Einrichtungen, das heißt, dass ab einer bestimmten Bevölkerungszahl die Tragfähigkeit für bestimmte Einrichtungen unterstellt wird. Ein kausaler Zusammenhang zwischen der Bevölkerung im Zentralen Ort selbst und der Tragfähigkeit seiner zentralörtlich bedeutsamen Infrastruktur besteht jedoch nicht. Neben der Bevölkerungszahl im Versorgungsbereich sind auch die Raumstruktur und damit einhergehend die Versorgungsalternativen (Mehrfachorientierung der Bereichsbevölkerung) von Bedeutung für die Bestimmung der Tragfähigkeit. Insofern sollte von pauschalen Einwohnerschwellenwerten sowohl als Ausweisungskriterium für Zentrale Orte als auch für die Abgrenzung der Versorgungsbereiche abgesehen werden.

Vielmehr ist eine raumstrukturelle Differenzierung sinnvoll und erscheint auch rechtlich geboten. So weist Spannowsky (in Spannowsky/Runkel/Goppel 2010, Kommentar zu $\S 2$ ROG, Rn. 77) darauf hin, dass es Aufgabe der Raumordnung sei, den „Gewährleistungsumfang hinsichtlich der Ausstattung der Bevölkerung mit Dienstleistungen sowie technischer und sozialer Infrastruktur unter Berücksichtigung der raumstrukturellen und raumfunktionellen Gegebenheiten zu bestimmen". Eine entsprechende Flexibilisierung ist bereits im ROG angelegt, wird aber bislang von den Ländern nur teilweise umgesetzt (Gleichwertigkeit der Lebensverhältnisse, $\S 1$ Abs. 2 i. V. m. $\S 2$ Abs. 2 Nr. 3 ROG: ,die Erreichbarkeits- und Tragfähigkeitskriterien des Zentrale-Orte-Konzepts sind flexibel an regionalen Erfordernissen auszurichten").

Für die Begründbarkeit zentralörtlicher Funktionszuweisungen sind die regionalen Gegebenheiten zunächst empirisch zu ermitteln. Aus dieser Analyse lässt sich dann ableiten, welche Einwohnerzahl als erforderlich gelten kann, um eine tragfähige Infrastruktur (ermittelt über das Vorhandensein der in Ausstattungskatalogen definierten Einrichtungen) zu unterhalten (vgl. auch Winkel/Greiving 2008).

Schließlich ist auch die Aufnahme von Ausnahmetatbeständen unter Wahrung der Systemgerechtigkeit erforderlich. So kommt etwa der Lage eines Zentralen Orts im Raum eine große Bedeutung zu, wenn es im Einzelfall zur Schließung von Netzlücken bei Gewährleistung einer zumutbaren Erreichbarkeit der Absenkung von Tragfähigkeitsstandards bedarf (z. B. Klassenteiler zur Bestimmung von Mindestschülerzahlen im Bildungswesen). In derartigen Fällen sind geeignete Orte zu identifizieren, die trotz vorhandener Ausstattungsdefizite normativ als Zentraler Ort festgelegt werden. Soll die Tragfähigkeit Priorität genießen, ist eine flexible Handhabung von Schwellenwerten zur Erreichbarkeit der Zentralen Orte zu gewährleisten. Dies kann die Entwicklung alternativer Anbindungsformen (z. B. Bürgerbusse) ebenso umfassen wie temporäre oder mobile Angebotsformen.

Infolgedessen ist klarzustellen, dass keine Gemeinde einen Anspruch auf eine zentralörtliche Funktionszuweisung alleine aus ihrer Ausstattung ableiten können sollte. Diese ist zwar eine zu erfüllende, aber als solche nicht hinreichende Voraussetzung für eine entsprechende Funktionszuweisung. Zusätzlich ist die raumordnerische Erforderlichkeit einer Funktionszuweisung im Einzelfall zu prüfen - vor dem Hintergrund eines Zentrale-Orte-Konzepts, das eine flächendeckende, auch unter prognostischen Gesichtspunkten langfristig tragfähige und erreichbare Versorgung und Entwicklung im Planungsraum zum Ziel hat. Insofern können und sollten im Einzelfall auch hinsichtlich ihrer Ausstattung gleiche Gemeinden ungleich behandelt bzw. eingestuft werden, ohne dass damit das Gebot der Systemgerechtigkeit oder der Gleichbehandlungsgrundsatz verletzt werden würden.

\subsection{Ausstattungskataloge}

Zentrale Einrichtungen zählen seit den Entschließungen der MKRO zu Zentralen Orten zum Wesenskern von Zentrale-Orte-Konzepten. Grundsätzlich gilt: Ausstattungskataloge (mit zentralen Einrichtungen) können normativer oder deskriptiver Art sein. Normative Kataloge im Sinne eines raumordnerischen Ziels nennen verbindliche Ausstattungskriterien, die der Zentrale Ort (i. d. R. eine Gemeinde) zur Einstufung in die entsprechende Stufe aufweisen muss bzw. die ihm zur vollen Funktionsausübung im Sinne eines planerisch angestrebten Sollzustands noch fehlen. Deskriptive Kataloge hingegen werden zur empirischen Untersuchung des Ist-Zustands eines Zentrale-Orte-Systems herangezogen 
- und können insofern auch als ergänzende Einstufungskriterien zur Anwendung kommen.

Die Festlegung verbindlicher Einrichtungen ist als Konkretisierung der Regelungen in $\S 2$ Abs. 2 S. 3 ROG zu verstehen: „Die Versorgung mit Dienstleistungen und Infrastrukturen der Daseinsvorsorge, insbesondere die Erreichbarkeit von Einrichtungen und Angeboten der Grundversorgung für alle Bevölkerungsgruppen, ist zur Sicherung von Chancengerechtigkeit in den Teilräumen in angemessener Weise zu gewährleisten; dies gilt auch in dünn besiedelten Regionen. Die soziale Infrastruktur ist vorrangig in Zentralen Orten zu bündeln; die Erreichbarkeitsund Tragfähigkeitskriterien des Zentrale-Orte-Konzepts sind flexibel an regionalen Erfordernissen auszurichten." Vor diesem Hintergrund sind es ausschließlich grundzentrale Einrichtungen, die normativ als Ziel der Raumordnung festzulegen sind.

Die Akademie für Raumforschung und Landesplanung (ARL) (Blotevogel 2002, S. 244, 296 f.) hat darauf hingewiesen, dass verbindliche (allerdings auch explizit ausdifferenzierte) Ausstattungskataloge im Sinne von Zielen der Raumordnung problematisch seien, weil die Gemeinden als Normadressaten einer mit der zentralörtlichen Einstufung einhergehenden Funktionszuweisung bestimmte, ihnen noch fehlende Infrastruktureinrichtungen einfordern können. Hier wird allerdings keineswegs für einen ausdifferenzierten Katalog plädiert, vielmehr sollten nur solche Einrichtungen als verbindliche Mindeststandards festgelegt werden, die einem logischen und politisch legitimierten Ziel-Mittel-System im Sinne des ROG in Verbindung mit dem jeweiligen Landesraumordnungsplan folgen.

Grundsätzlich und unabhängig von der Hierarchieebene sollten nur solche Güter oder Einrichtungen Bestandteil von Zentrale-Orte-Konzepten sein, die erstens nicht ubiquitär im Raum vorgehalten werden bzw. im Sinne der Erschließungspflicht vorgehalten werden müssen, die zweitens auf den Endverbraucher ausgerichtet sind (z. B. haushaltsorientierte im Gegensatz zu unternehmensorientierten Dienstleistungen) und die drittens sachlich bestimmt (z. B. Arbeitsgericht) oder zumindest bestimmbar sind (z. B. Gerichte unterer Instanz). Nicht einbezogen werden sollte die metropolitane Ebene: Sie hat keine ordnungsrechtliche Relevanz. Zudem geht es auf dieser Ebene nicht primär um Einrichtungen, die der Versorgung der Bevölkerung dienen (Versorgungsfunktion Zentraler Orte), sondern um (hochrangige) Entwicklungsfunktionen.

\subsection{Zentralörtliche Versorgungsbereiche}

Zentralörtliche Versorgungsbereiche sind wesentlicher Bestandteil der Zentrale-Orte-Konzepte in den Ländern, wobei teilweise nicht für alle Hierarchiestufen Bereiche festgelegt sind und in Einzelfällen lediglich Hinweise zu ihrer Abgrenzung gegeben werden. Bedeutsam sind zentralörtliche Versorgungsbereiche gegenwärtig insbesondere hinsichtlich der Bestimmbarkeit von Zielen der Raumordnung im Zusammenhang mit dem Kongruenzgebot bzw. Beeinträchtigungsverbot (Steuerung des großflächigen Einzelhandels, vgl. Uechtritz 2011) und für den kommunalen Finanzausgleich, sofern sich die „Einwohnerveredelung“ (das heißt der einwohnerbezogene Zuschlag für zentralörtliche Funktionen bei den Schlüsselzuweisungen) wie in Rheinland-Pfalz auf die Versorgungsbereiche bezieht. Zudem machen sich Dritte wie die Kassenärztliche Bundesvereinigung die definierten Versorgungsbereiche zu eigen. Für die ambulante medizinische Versorgung und damit in einem zentralen Bereich der Daseinsvorsorge entfalten Zentrale-Orte-Konzepte gemäß $\S 11$ Abs. 3 BPl-RL über die Abgrenzung von Mittelbereichen eine weitreichende Steuerungswirkung (Gemeinsamer Bundesausschuss 2013). Insofern ist die Auffassung der ARL von 2002, den Versorgungsbereichen solle lediglich ein Erläuterungscharakter zukommen (Blotevogel 2002, S. 300), überholt.

Die grundsätzliche Festlegung zentralörtlicher Versorgungsbereiche ist wiederum - theorie- und systemimmanent - für Zentrale-Orte-Konzepte erforderlich: Erstens resultiert die Zentralität eines Ortes meist aus seiner Umlandbedeutung („Mitversorgung"). Davon ausgenommen sind lediglich sogenannte „Selbstversorgerorte“, wobei diese streng genommen ebenfalls per Definition mit ihrem Gemeindeterritorium einen Versorgungsbereich aufweisen. Zweitens sind allein Hinweise zur Abgrenzung nicht ausreichend, da damit bestenfalls das zentralörtliche System (deskriptive Verflechtungsbereiche) beschrieben werden kann, ohne dass damit politisch-planerische Aussagen verknüpft wären. Den Ausführungen des OVG Lüneburg (1 KN 152/10 vom 15.03.2012), wonach die Angabe einer Methodik ausreichend sei, ist insofern zu widersprechen, als dies zwar für monothematische Zwecke (z. B. Einzelhandelssteuerung) sachgerecht sein mag, nicht jedoch für multifunktionale Zwecke, die mit Zentrale-Orte-Konzepten und damit den zentralörtlichen Versorgungsbereichen zweifelsohne verknüpft sind.

Zwar ist die Bestimmbarkeit eines Ziels der Raumordnung (etwa durch Angabe von Kriterien, Ermittlungsmethoden usw.) grundsätzlich gleichwertig zur (räumlichen) Bestimmung. Die Festlegung Zentraler Orte begründet sich allerdings primär über ihre Versorgungsaufgaben für Dritte im Rahmen eines raumordnerischen Gesamtkonzepts: nur bereichsbildende Gemeinden sollten als Zentrale Orte festgelegt werden. Schließlich ist die normative Abgrenzung durch den Plangeber auch dann erforderlich, wenn an die Festlegung Rechtsfolgen etwa im Rahmen der Einzelhandelssteuerung, des kommunalen Finanzausgleichs oder bei der Etablierung von Verantwortungsgemeinschaften (Kooperationserfordernis) geknüpft sind. 
Die stark voneinander abweichenden Schwellenwerte unter den Bundesländern sind als solche insofern nicht problematisch, da sie potenziell auch Ausdruck unterschiedlicher Raumstrukturen, aber auch planungspolitischer Ziele sein können. Fragwürdig sind derartige Unterschiede erst dann, wenn - wie in etlichen Bundesländern - keine empirische Herleitung zu ihrer Begründung herangezogen werden kann oder sie sich nicht aus den Rechtsnormen erschließen. Die relevanten Tragfähigkeitsschwellenwerte für die Versorgungsbereiche können allerdings nur dann empirisch begründet hergeleitet werden, wenn ein politisch legitimiertes Zielsystem von Erreichbarkeitsstandards die Dimensionierung und somit die Abgrenzung der Versorgungsbereiche ermöglicht.

Methodisch bietet es sich zur Ermittlung der Bereichsbildungsfähigkeit von Gemeinden an, anhand eines Anbindungskoeffizienten (ermittelt über Arbeitsmarkt- und Schülerpendler) all jene Quellorte einem (dann bereichsbildenden) Zielort zuzuordnen, zu dem erstens der stärkste Anbindungskoeffizient besteht (Zielort) und der zweitens eine höhere rangstufenspezifische Versorgungsleistung (Gravitationskraft) aufweist als der Quellort (Terfrüchte 2015, S. 173). Es können also auch jene unterdurchschnittlich ausgestatteten Zielorte zunächst bereichsbildend wirken und als potenzielle Zentrale Orte in Frage kommen, die mangels erreichbarer und besser ausgestatteter Alternativen innerhalb eines Teilraums noch die vergleichsweise stärksten Orte sind. Erforderlich für die Bestimmung Zentraler Orte ist daher neben der Bereichsbildungsfähigkeit die Lokalisierung der vom Plangeber als - für die jeweilige Hierarchiestufe - verbindlich festgelegten Funktionen bzw. Einrichtungen. Von den empirisch ermittelten Anbindungen kann normativ abgewichen werden, wenn

- die Erreichbarkeit eines Zielortes von einem Quellort aus nicht zumutbar erscheint (wofür operationalisierte Erreichbarkeitsschwellen unverzichtbar sind). In diesem Fall ist eine Zuordnung zu einem Versorgungsbereich eines anderen Zentralen Ortes opportun, zu dem zwar nicht die stärkste, aber eine signifikante Anbindung besteht.

- der Zielort sich in einem anderen Gemeindeverband (grundzentrale Ebene), anderen Landkreis (mittelzentrale Ebene) oder einer anderen Planungsregion (oberzentrale Ebene) als der Quellort befindet. Hier kann zur Wahrung der Verwaltungskongruenz die Zuordnung zu einem anderen Versorgungsbereich sinnvoll sein, zu dem zwar nicht die stärkste, aber eine signifikante Anbindung besteht.

- Gegebenenfalls kann sogar die Ausweisung eines nicht bereichsbildenden Ortes als Zentraler Ort erforderlich werden, wenn ansonsten keine Erreichbarkeit gewährleistet werden könnte. In diesem Fall bestünde aber ein strukturpolitisches Stärkungserfordernis. Alternativ wäre von der Erreichbarkeitsschwelle zugunsten der Tragfähigkeit abzuweichen.

Folglich ist die Einstufung Zentraler Orte im Einzelfall nur nachvollziehbar und damit begründbar, wenn ihnen entsprechende Versorgungsbereiche zugeordnet werden - entweder zeichnerisch in einer Festlegungskarte oder aber zumindest über Gemeindelisten und ihre Zuordnung zu einem höherrangigen Zentralen Ort. Damit ist dann auch die Ungleichbehandlung ausstattungsgleicher Gemeinden begründbar, etwa weil ihre Festlegung aus Gründen der Erreichbarkeit im Einzelfall erforderlich ist.

\section{Prozedurale Empfehlungen zur Aufstellung bzw. Fortschreibung von Zentrale-Orte-Konzepten}

Es ist dringend anzuraten, in der Begründung zu den entsprechenden Zielen und/oder Grundsätzen, die das ZentraleOrte-Konzept eines Landes leiten, transparent darzulegen, welche Analysemethode - etwa zur Abgrenzung zentralörtlicher Bereiche - aus welchen Gründen verwendet wurde. Dies begründet sich mit den rechtlichen Steuerungswirkungen, die von den Zentrale-Orte-Konzepten in vielen Bundesländern in den Bereichen Steuerung des großflächigen Einzelhandels, kommunaler Finanzausgleich und Siedlungsflächenentwicklung ausgehen. Zudem sind konsistente Zentrale-Orte-Konzepte von wesentlicher Bedeutung für die Sicherung der Daseinsvorsorge: Nur unter der Voraussetzung, dass Fachplanungsträger sich ein Zentrale-Orte-Konzept aufgrund seiner Plausibilität zu eigen machen, werden sie sich auch daran orientieren, da die formal bestehende allgemeine Raumordnungsklausel des $\S 4$ Abs. 1 ROG, die öffentliche Planungsträger an die Ziele der Raumordnung bindet, faktisch nicht wirksam ist.

Die gängige Praxis ist in vielen Bundesländern jedoch eine andere: Wenn normative Setzungen überhaupt (empirisch) begründet werden, wird regelmäßig nicht klar gemacht, welche Annahmen getroffen wurden und welche Methodik zur Anwendung kam. Verweise auf Erläuterungstexte oder externe Gutachten reichen nicht aus, um Ziele der Raumordnung zu begründen, weil Erläuterungen oder externe Gutachten nicht an der Bindungswirkung der Ziele der Raumordnung teilnehmen. Will sich ein Planungsträger ein Gutachten zu eigen machen, muss er dessen zentrale Annahmen und die verwendete Methodik in der Begründung offenlegen, um der erforderlichen Normklarheit bzw. der Forderung der Verständlichkeit des Planwerks aus sich selbst heraus Genüge zu tun.

Aus diesen Überlegungen heraus lässt sich ein idealtypischer Prozess einer Neuaufstellung bzw. Fortschreibung 
eines Zentrale-Orte-Konzepts durch Landes- und/oder Regionalplanungen beschreiben:

\subsection{Entwicklung eines Zielsystems (normative Wertebene)}

Zunächst sollte ein Zielsystem entwickelt werden, das als Grundlage für eine empirische Prüfung des bestehenden Zentrale-Orte-Systems dient. Aus diesem Zielsystem lässt sich nachvollziehen, ob spätere Einstufungen im Zentrale-Orte-Konzept „systemgerecht“ vorgenommen werden. Das Zielsystem sollte durch ein Regel-Ausnahme-Verhältnis gekennzeichnet sein und auch Regeln für die Kollision von Rechtsnormen vorsehen. Dies gilt insbesondere für das Spannungsverhältnis zwischen Tragfähigkeit und Erreichbarkeit: Es ist klarzustellen, ob im Kollisionsfall der Erreichbarkeit Vorrang einzuräumen ist (mit der Konsequenz, dass nicht tragfähige Einrichtungen aufrechterhalten werden müssen und für diese beispielsweise in Abstimmung mit der Schulentwicklungsplanung im Bildungswesen andere Klassenteiler festgelegt werden oder temporäre bzw. räumlich flexible Angebote geschaffen werden) oder ob Erreichbarkeiten flexibilisiert werden. Wesentliche Bestandteile dieses Zielsystems sind:

- Festlegungen zur Steuerungswirkung (z. B. für Bereiche wie Siedlungsflächenentwicklung, Daseinsvorsorge, großflächiger Einzelhandel, kommunaler Finanzausgleich),

- Festlegungen zu Hierarchiestufen und gegebenenfalls Kooperationserfordernissen für Mehrfachorte,

- Festlegungen zu Tragfähigkeit und Erreichbarkeit (und Kollisionsregeln für den Fall von deren Unvereinbarkeit), die bei heterogenen Raumstrukturen zu differenzieren sind,

- Festlegungen zur Bereichsabgrenzung und eine Erläuterung der verwendeten Methodik sowie

- Festlegungen zu erforderlichen Ausstattungen (getrennt in verbindliche und empfohlene Einrichtungen).

Das Zielsystem sollte in enger Abstimmung mit den Adressaten des Zentrale-Orte-Konzepts und hier insbesondere den Fachplanungen entwickelt werden. Es handelt sich bei seiner Entwicklung nicht um einen streng linearen Prozess, weil Schwellenwerte oder - landes- bzw. regionalspezifische - Ausstattungsmerkmale erst aus der Empirie abgeleitet und dann in das Zielsystem aufgenommen werden können.

\subsection{Empirische Überprüfung des Zentrale-Orte-Systems}

Es ist mithilfe der gewählten Untersuchungsmethodik das empirisch beschreibbare Zentrale-Orte-System abzubilden, das die bestehenden räumlich-funktionalen Verflechtungen und zentralörtlichen Funktionen der Gemeinden analy- siert und beschreibt. Wichtig ist dabei, dass der empirische Befund zwar die planerischen Entscheidungen und Festlegungen vorbereiten sollte, diese jedoch nicht ersetzen kann. Prinzipiell ist die Frage der Auswahl einer geeigneten Methodik von der Diskussion geeigneter Kriterien zu trennen, das heißt, es können unabhängig von der gewählten Methodik durchaus die gleichen Kriterien Verwendung finden. Die Kriterien zur Ausstattung und Ausweisung Zentraler Orte (und auch eine mögliche Gewichtung) müssen sich auf das politisch legitimierte Zielsystem zurückführen lassen und können vom Gutachter nicht frei gewählt werden. Gleichwohl kann sich während der empirischen Überprüfung das Erfordernis einer Anpassung des Zielsystems abzeichnen; etwa wenn deutlich wird, dass im Zielsystem definierte Ausstattungskriterien nicht länger rangstufenüblich sind. Insofern handelt es sich um einen iterativen Prozess.

Die jeweiligen methodischen Ansätze können also der Komplexitätsreduzierung auf der Sachebene dienen und das politische Entscheidungsproblem auf diejenigen Fragen fokussieren, die der Wertebene zuzurechnen sind. Die einzig richtige Methode gibt es nicht, sondern nur eine Reihe mehr oder weniger zweckmäßiger Vorgehensweisen, wobei sich die Zweckmäßigkeit erst im Einzelfall aus den gegebenen Rahmenbedingungen heraus beurteilen lässt.

\subsection{Die Anwendung des Zielsystems auf der Ebene des Sachmodells}

Zunächst gilt es, zentralörtliche Verflechtungsbereiche abzugrenzen und die Zentralen Orte innerhalb der Bereiche entsprechend ihrer räumlichen Lage (unter anderem im Hinblick auf Erreichbarkeitsstandards), der zu versorgenden Bevölkerung im Bereich, ihrer funktionalen Ausstattung und der Potenziale für die Versorgung ihrer Bevölkerung und ihrer künftigen Entwicklung einer Hierarchiestufe zuzuordnen. Atypische Einzelfälle (z. B. im Zusammenhang mit ausgeprägten grenzüberschreitenden Verflechtungen, besonderen topographischen Verhältnissen wie Gebirgszüge oder Seen usw.) sind besonders zu begründen. Für die Entwicklung des normativen Konzepts kann auch vom empirischen Befund (Sachebene) abgewichen werden, sofern sich diese Abweichung aus dem politisch legitimierten Zielsystem (Wertebene) herleiten lässt (z. B. Stärkung eines Orts zur Sicherung der Erreichbarkeit). Üblicherweise sollten die normativen Konzepte daher auch vom empirisch beschreibbaren System abweichen, sofern nicht ausschließlich Bestandswahrung erfolgen soll.

Insofern ist im Raumordnungsplan begrifflich deutlich zu trennen zwischen dem empirisch beschreibbaren Zentrale-Orte-System und dem normativen raumordnerischen Konzept. Es wird empfohlen, sowohl das Ergebnis der empirischen Überprüfung als auch die Anwendung des 
Zielsystems räumlich in Kartenform darzustellen, um die Systemgerechtigkeit des Konzepts transparent und nachvollziehbar zu machen. Während das Konzept Teil einer Festlegungskarte ist, sollte das Zentrale-Orte-System in einer Erläuterungskarte dargestellt werden. In der Erläuterungskarte könnten etwa Mehrfachausrichtungen bei den zentralörtlichen Verflechtungsbereichen dargestellt werden, wohingegen in der Festlegungskarte i. d. R. eine eindeutige Zuordnung zu Versorgungsbereichen im Sinne räumlicher Bestimmtheit erfolgen sollte. Da die Gemeinden Funktionsempfänger sind, sollten auch eindeutige, aber Gemeindegrenzen durchschneidende Verflechtungsbereiche (sofern diese empirisch ermittelt werden) an die tatsächlichen Gemeindegrenzen approximiert werden; dieser normative Schritt führt ebenfalls zu vom Befund abweichenden Grenzen von Versorgungsbereichen. Auf diese Weise würden die Funktionszuweisungen in den Zentrale-Orte-Konzepten der Länder nachvollziehbarer und damit auch verständlicher und letztendlich überzeugender, womit ihre Steuerungswirkung und die Akzeptanz (vor allem seitens der Fachplanungen und Fachpolitiken) gestärkt werden könnte.

\section{Fazit}

Der vorliegende Beitrag hat einen umfassenden Überblick über den Stand der Zentrale-Orte-Konzepte in der Raumordnungspraxis der Länder geliefert. Deutlich wurde, wie heterogen die unterschiedlichen Konzepte sind, was deren Vergleichbarkeit enorm erschwert. Differenzierte Zielsysteme mit raumstrukturell unterschiedlichen Schwellenwerten sollten aber nicht in Frage gestellt werden, insoweit sie sich aus unterschiedlichen Steuerungserfordernissen heraus begründen lassen. Es konnten dennoch eine Reihe von Ansätzen zur Weiterentwicklung von Zentrale-OrteKonzepten ermittelt werden, die jenseits der zu Recht differierenden Zielsysteme für alle Bundesländer Gültigkeit besitzen. Deutlich geworden ist vor allem, wie wesentlich erstens ein nachvollziehbares empirisches Vorgehen zur Bestimmung Zentraler Orte und ihrer Versorgungsbereiche und zweitens eine klare Trennung zwischen dem empirischen Befund und dem normativen Konzept sind. Gerade die - begründete - Abweichung vom Befund macht den raumordnerischen Steuerungswillen aus. Zweifellos ist jede evidenzbasierte Fortentwicklung eines Zentrale-Orte-Konzepts ein planungspolitischer Kraftakt, der aber angegangen werden sollte, da nur so die Gerichtsfestigkeit der Konzepte und ihre faktische Steuerungswirkung für Dritte gewährleistet werden können. Insofern ist mit jeder Fortentwicklung immer auch ein enormer Kommunikationsaufwand verbunden - sowohl verwaltungs- bzw. regierungsintern als auch gegenüber Kommunen und Fachplanungen.
Ein erstes Handlungserfordernis, welches praktisch für alle Flächenländer gilt, ist die Abkehr von (starren) Ausstattungs- und Tragfähigkeitsstandards als wesentliche Ausweisungskriterien. Ein zweites betrifft die raumstrukturell differenzierte Betrachtung, wozu jedoch bewusst keine weitergehenden Empfehlungen zur situationsadäquaten Handhabung der Zentrale-Orte-Konzepte vorgelegt werden. Die jüngere wissenschaftliche Diskussion um Strukturvarianten im Zentrale-Orte-Konzept wie Städteverbünde und Funktionsräume (vgl. etwa Winkel/Greiving 2008) zeigt, dass zur Operationalisierung dieser Ansätze noch ein erheblicher Forschungsbedarf besteht, dessen Darlegung den Rahmen dieses Beitrags ebenso sprengen würde wie vergleichende Untersuchungen und Empfehlungen zur Steuerungswirkung der Zentrale-Orte-Konzepte.

\section{Literatur}

Bartholomae, F. W.; Schoenberg, A. M. (2013): Kann das ZentraleOrte-System die flächendeckende Daseinsvorsorge in Bayern gewährleisten? In: InfrastrukturRecht, 10 (11), 316-319 (Sonderausgabe „Kommunales Infrastruktur-Management“).

Bartram, G. (2010): Die Ziele der Raumordnung. Ein Planungsinstrument im Spannungsfeld zwischen gewachsenem Steuerungsanspruch und verfassungsrechtlichen Anforderungen. Baden-Baden. $=$ Studien zu Staat, Recht und Verwaltung, Bd. 20.

Bartsch, R. (2006): Funktionsteilige zentrale Orte in Deutschland. Zur Umsetzung interkommunaler Zusammenarbeit im zentralörtlichen Verbund. Jena. = Schriftenreihe Wirtschaftsgeographie + Regionalentwicklung, Bd. 5.

Blotevogel, H. H. (Hrsg.) (2002): Fortentwicklung des Zentrale-Orte-Konzepts. Hannover. $=$ Forschungs- und Sitzungsberichte der Akademie für Raumforschung und Landesplanung, Bd. 217.

BMVBS - Bundesministerium für Verkehr, Bau und Stadtentwicklung (Hrsg.) (2008): Kooperation Zentraler Orte in schrumpfenden Regionen. Bonn. $=$ Werkstatt: Praxis 53 .

BMVBS - Bundesministerium für Verkehr, Bau und Stadtentwicklung (Hrsg.) (2010): Sicherung der Daseinsvorsorge und Zentrale-Orte-Konzepte - gesellschaftspolitische Ziele und räumliche Organisation in der Diskussion. Bonn. = BMVBS-Online-Publikation, Nr. 12/2010.

Greiving, S. (2006): Das raumordnerische Konzept der Städteverbünde und seine Umsetzung in der landesplanerischen Praxis. In: Raumforschung und Raumordnung, 64 (1), 5-17.

MKRO - Ministerkonferenz für Raumordnung (1968): Zentrale Orte und ihre Verflechtungsbereiche. Entschließung der Ministerkonferenz für Raumordnung vom 8. Februar 1968. Abgedruckt in der BT-Drucksache V/3958, S. 149. http://dipbt.bundestag.de/doc/ btd/05/039/0503958.pdf (24.06.2015).

MKRO - Ministerkonferenz für Raumordnung (1972): Zentralörtliche Verflechtungsbereiche mittlerer Stufe in der Bundesrepublik Deutschland. Entschließung der Ministerkonferenz für Raumordnung vom 15. Juni 1972. Abgedruckt in der BTDrucksache VI/3793, S. 146-147. http://dipbt.bundestag.de/doc/ btd/06/037/0603793.pdf (24.06.2015).

MKRO - Ministerkonferenz für Raumordnung (1983): Oberzentren. Entschließung der Ministerkonferenz für Raumordnung vom 16. Juni 1983. Bonn. 
MKRO - Ministerkonferenz für Raumordnung (2006): Leitbilder und Handlungsstrategien für die Raumentwicklung in Deutschland. Beschluss vom 30.06.2006. Berlin.

MKRO - Ministerkonferenz für Raumordnung (2008): Eckpunktebeschluss „Demographischer Wandel und Daseinsvorsorge“ vom 29. April 2008. Berlin. http://www.bmvi.de/SharedDocs/DE/Anlage/ StadtUndLand/LaendlicherRaum/eckpunktebeschlus-zum-demographischen-wandel-und-daseinsvorsorge-29-april-2008.pdf? blob=publicationFile (26.06.2015).

MKRO - Ministerkonferenz für Raumordnung (2013): Leitbilder und Handlungsstrategien für die Raumentwicklung in Deutschland 2013. MKRO-Beschluss vom 3. Juni 2013. Berlin.

Spannowsky, W. (2011): Forschungsprojekt: Konkretisierung der Grundsätze der Raumordnung durch die Bundesraumordnung. Endbericht. http://www.bbsr.bund.de/BBSR/DE/FP/ReFo/ Raumordnung/2010/Grundsaetze/Download_Handbuch.pdf? blob $=$ publicationFile \& $=2(19.08 .2013)$.

Spannowsky, W.; Runkel, P.; Goppel, K. (2010): Raumordnungsgesetz (ROG) Kommentar. München.

Terfrüchte, T. (2015): Regionale Handlungsräume. Gliederung und Einflussfaktoren am Beispiel Nordrhein-Westfalens. Detmold. = Metropolis und Region 14.

Uechtritz, M. (2011): Neues zur raumordnerischen Einzelhandelssteuerung: Zulässigkeit von Kongruenzgeboten als Soll-Ziele zugleich Anmerkung zu BVerwG, Urt. v. 16.12.2010 - 4 C 8.10. In: ZfBR 34 (7), 648-656.

Winkel, R.; Greiving, S. (2008): Zentrale-Orte-Konzepte in praxisorientierter Weiterentwicklung. Monozentrale Orte, Städteverbünde und Zentralörtliche Funktionsräume In: RaumPlanung, 141, 263-268.

\section{Rechtsquellenverzeichnis}

Bayerische Staatsregierung (2013): Landesentwicklungsprogramm Bayern. LEP 2013, vom 22.08.2013. Fundstelle: GVB1 2013, S. 550.

BVerwG, Urteil vom 16.12.2010 - Aktenzeichen 4 C 8.10 10. http://www.bverwg.de/entscheidungen/entscheidung. php?ent=161210U4C8.10.0 (26.06.2015).

Gemeinsamer Bundesausschuss (G-BA) (Hrsg.) (2013): Richtlinie des Gemeinsames Bundesausschusses über die Bedarfsplanung sowie die Maßstäbe zur Feststellung von Überversorgung und Unterversorgung in der vertragsärztlichen Versorgung - Bedarfsplanungs-Richtlinie (BPl-RL) - Neufassung vom 20. Dezember 2012, zuletzt geändert am 20. Juni 2013. BAnz AT 29.07.2013 B3.

Gesetz zu dem Staatsvertrag der Länder Berlin und Brandenburg über das Landesentwicklungsprogramm 2007 (LEPro 2007) und die Änderung des Landesplanungsvertrages vom 18. Dezember 2007. Fundstelle: GVB1. I/07, S. 235
Hessische Landesregierung (2000): Landesentwicklungsplan. LEP 2000, vom 12.01.2003. Fundstelle: GVB1. 2001 I, S. 2.

Landesplanungsgesetz Schleswig-Holstein (LaplaG) in der Fassung der Bekanntmachung vom 10. Februar 1996 (GVOBl. 1996, S. 232), zuletzt geändert durch Gesetz vom 22.05.2015 (GVOB1., S. 132).

Landesregierung Baden-Württemberg (2002): Landesentwicklungsplan 2002 Baden-Württemberg. LEP 2002, vom 23.07.2002. Fundstelle: GBl. Nr. 9, S. 301.

Landesregierung Mecklenburg-Vorpommern (2005): Landesraumentwicklungsprogramm Mecklenburg-Vorpommern. LEP 2005, vom 30.05.2005. Fundstelle: Amtsbl. M-V 2005, S. 797.

Landesregierung Niedersachsen (2012): Landes-Raumordnungsprogramm Niedersachsen. LROP, vom 24.09.2012. Fundstelle: Nds. MB1. Nr. 20/2012, S. 350

Landesregierung Nordrhein-Westfalen (2013): Landesentwicklungsplan Nordrhein-Westfalen Entwurf. LEP Entwurf 2013, vom 25.06.2013. Fundstelle: MB1. NRW. 2013, S. 312.

Landesregierung Sachsen-Anhalt (2011): Landesentwicklungsplan 2010. LEP 2010, vom 16.02.2011. Fundstelle: GVB1. LSA 2011, S. 160.

Landesregierung Thüringen (2014): Landesentwicklungsprogramm Thüringen 2025. LEP 2025, vom 15.05.2014. Fundstelle: TH GvBl. 2014, Nr. 6, S. 205-381.

LEP S-H (2010): Innenministerium Schleswig-Holstein: Landesentwicklungsplan Schleswig-Holstein 2010 vom 13.07.2010. Fundstelle: Amtsbl. Schl.-H., S. 719.

Ministerrat Rheinland-Pfalz (2008): Landesentwicklungsprogramm. LEP IV, vom 07.10.2008. Fundstelle: RP GVB1. Nr. 16, S. 5001-5290.

OVG Lüneburg, Urteil vom 15.03.2012 - Aktenzeichen 1 KN 152/10. http://www.rechtsprechung.niedersachsen.de/jportal/portal/page/ bsndprod.psml?doc.id=MWRE120001575\&st=null\&showdocca$\mathrm{se}=1(26.06 .2015)$.

ROG - Raumordnungsgesetz in der Fassung der Bekanntmachung vom 22. Dezember 2008 (BGB1. I, S. 2986), zuletzt geändert durch Artikel 9 des Gesetzes vom 31. Juli 2009. Fundstelle: BGB1. I, S. 2585.

Regierung des Saarlandes (2006): Landesentwicklungsplan, Teilabschnitt „Siedlung“. LEP 2006, vom 04.07.2006. Fundstelle: Amtsblatt des Saarlandes, Nr. 29, G1260.

Sächsische Staatsregierung (2013): Landesentwicklungsplan 2013. LEP 2013. Fundstelle: SächsGVB1. 11/2013, S. 582.

Senat von Berlin (2009): Landesentwicklungsplan Berlin-Brandenburg. LEP B-B, vom 31.03.2009. Fundstelle: GVB1., S. 182.

VerfGH NRW, Urteil vom 26.08.2009 - Aktenzeichen VerfGH 18/08. www.vgh.nrw.de/entscheidungen/2009/090826 18-08.pdf (26.06.2015). 